\title{
Proposal for Systemic-Therapy Response-Assessment Criteria at the Time of PSMA PET/CT Imaging: The PSMA PET Progression Criteria
}

\author{
Stefano Fanti ${ }^{1}$, Boris Hadaschik ${ }^{2,3}$, and Ken Herrmann ${ }^{3,4}$ \\ ${ }^{I}$ Nuclear Medicine, S. Orsola Hospital, University of Bologna, Bologna, Italy; ${ }^{2}$ Department of Urology, Essen University Hospital, \\ University of Duisburg-Essen, Essen, Germany; ${ }^{3}$ German Cancer Consortium, Heidelberg, Germany; and ${ }^{4}$ Department of Nuclear \\ Medicine, Essen University Hospital, University of Duisburg-Essen, Essen, Germany
}

\begin{abstract}
In around $20 \%$ of men with prostate cancer, metastasis develops during the course of their disease. Accordingly, discovering and developing new potent treatment strategies for patients with metastatic prostate cancer has been a major research focus during the last few decades. Identifying disease progression, especially within clinical trials, is essential in determining drug effectiveness. One major remaining question is how best to define disease progression. The criteria of the Prostate Cancer Clinical Trials Working Group (PCWG2) include clinical and laboratory parameters, as well as conventional imaging modalities such as MRI, CT, and bone scan findings, but advanced molecular imaging techniques, especially prostate-specific membrane antigen (PSMA) PET findings, are not considered. This is a problem because PSMA PET is used not only for detecting biochemical recurrence but also for restaging and as an intermediate-endpoint biomarker in ongoing clinical trials. Therefore, response criteria and PSMA PET progression (PPP) criteria need to be established with some urgency. The intent of this article is therefore to define prostate cancer progression by PSMA PET criteria. Our PPP proposal is based on the same principles as were applied for the PCGW2 criteria but adds value by including PSMA PET criteria. PPP defines PSMA treatment response using 3 different criteria. The first is the appearance of 2 or more new PSMA-positive distant lesions. The second is the appearance of 1 new PSMA-positive lesion plus consistent clinical or laboratory data and recommended confirmation by biopsy or correlative imaging within 3 mo of PSMA PET. The third is an increase in size or PSMA uptake of 1 or more existing lesions by at least $30 \%$, plus consistent clinical or laboratory data or confirmation by biopsy or correlative imaging within 3 mo of PSMA PET.
\end{abstract}

Key Words: prostate cancer; PSMA; PET; progression; 3P

J Nucl Med 2020; 61:678-682

DOI: $10.2967 /$ jnumed.119.233817

\section{$\mathbf{P}$} approximately $20 \%$ of patients will eventually succumb to metastatic disease. Accordingly, discovering and developing new potent

Received Jul. 12, 2019; revision accepted Nov. 11, 2019.

For correspondence or reprints contact: Ken Herrmann, Department of Nuclear Medicine, Universitätsklinikum Essen, Hufelandstrasse 55, 45147 Essen, Germany.

E-mail: ken.herrmann@uk-essen.de

Published online Dec. 5, 2019.

COPYRIGHT (C 2020 by the Society of Nuclear Medicine and Molecular Imaging. treatment strategies for metastatic prostate cancer has been a major research focus of the last few decades. Differentiating between responding and nonresponding patients, especially within clinical trials, is absolutely essential in determining drug effectiveness. The key challenge is to establish robust criteria for disease progression.

RECIST was long the mainstay for assessing treatment response in clinical trials involving patients with metastatic prostate cancer. This changed in 2008 when Scher et al. introduced the response criteria of the Prostate Cancer Clinical Trials Working Group (PCWG2) (1), which additionally included bone scan findings, and RECIST was no longer the mainstay. The most recent Prostate Cancer Working Group 3 (PCWG3) update, published in 2016, remained in essence unchanged (2). Prostate-specific membrane antigen (PSMA) PET has still not been incorporated.

PET imaging targeting PSMA has a major impact on prostate cancer staging and management $(3,4)$. It is now widely available in many countries and has been incorporated into major clinical guidelines (those of the European Association of Urology and National Comprehensive Cancer Network since 2017), especially for the evaluation of biochemical recurrence. Criteria defining a positive PSMA PET result (5-8) have been proposed. However, disease progression by PSMA PET has not been defined.

There is a need for well-defined progression criteria as patients, their families, and their physicians request unequivocal diagnostic information from PSMA PET studies. Moreover, drug companies are starting to integrate PSMA imaging into clinical trials and thus require firm definitions of progressive disease. The intent of this article is therefore to define prostate cancer progression by PSMA PET. The PSMA PET progression (PPP) proposal defines treatment responses by PSMA PET criteria taking into account the same principles as were applied for the PCGW2 criteria. We propose a validation strategy that is to be considered a starting point for further discussion and is to be updated as future data are published.

\section{PROPOSAL}

Progressive metastatic prostate cancer is usually defined by the appearance of new lesions or growth of existing lesions. The location of new lesions, local versus distant, may add prognostic relevance. Whereas the appearance of new lesions is rather easy to assess, an increase in the size or tracer uptake of existing lesions is more challenging. Regarding the definition of local and distant lesions, PPP focuses on the clinical impact, aggregating progression within the prostate, prostate bed, local recurrence, and pelvic lymph nodes as local. 
PPP takes into account the appearance of new lesions, their location, their size, and the intensity of tracer uptake to arrive at simple, robust, and reproducible criteria for disease progression in patients with metastatic prostate cancer. PPP builds on criteria established by PCWG2 that defined bone progression as the appearance of at least 2 new lesions on radionuclide bone scans. However, PPP also requires 2 or more additional new lesions identified on a confirmatory scan within at least 6-9 wk, resulting in the term $2+2$ rule. This delay in time to differentiate between flare and true progression may not be needed with PET imaging.

Two new distant lesions are required to fulfill the definition of disease progression (Fig. 1). This approach was chosen to reduce the risk of false-positives, including the flare phenomenon. However, because the flare phenomenon is comparatively uncommon for PSMA PET, and false-positive findings rarely occur in pairs, the proposed PPP concept does not require a confirmatory scan (9). In cases of multiple lesions with discordant behavior, that is, when some lesions are responding by size or uptake parameters or are even disappearing while others are newly appearing, the same criteria apply. Therefore, the emergence of 2 new distant lesions independent of discordant treatment-response behavior by existing lesions meets the criteria for progression (Figs. 2 and 3).

The appearance of only 1 new lesion is not sufficient for progression according to PCGW2, as is justified by the poor specificity of bone scanning (Fig. 4). In contrast, because of its high specificity, 1 new distant lesion by PSMA PET is considered progressive disease by PPP (Fig. 3) if the following criteria are met: consistent clinical or laboratory data, including prostate-specific antigen and other parameters such as pain assessment, lactate dehydrogenase, and anemia. Lesion validation by either biopsy or correlative imaging within 3

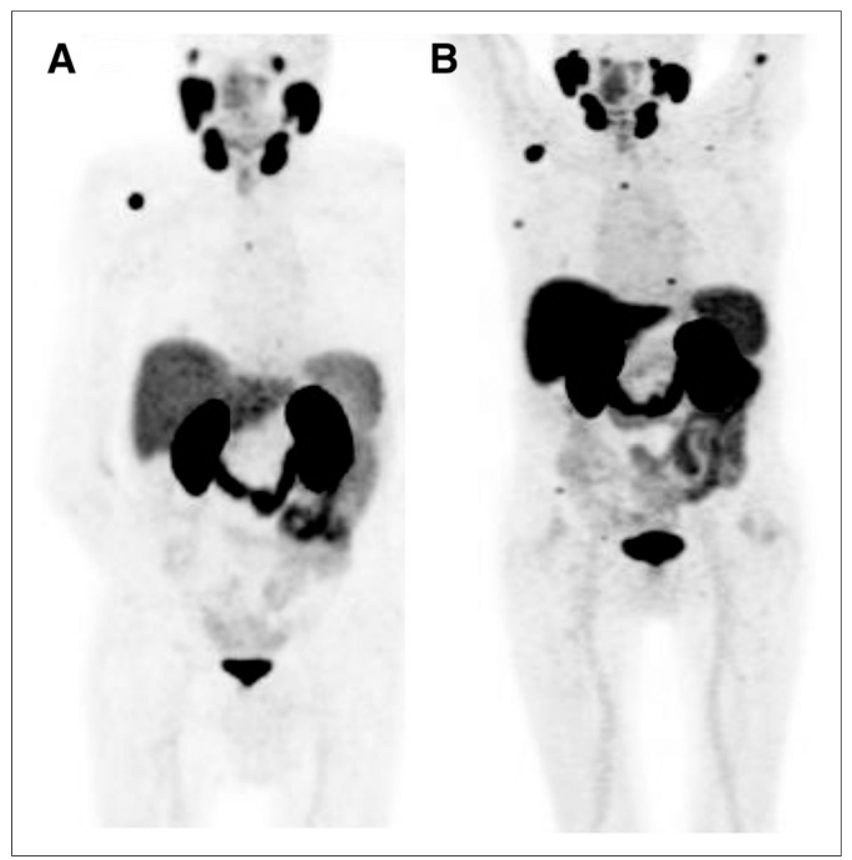

FIGURE 1. PPP for appearance of more than 2 new distant lesions in 77-y-old patient after radical prostatectomy (pT3bpNO Mx; Gleason score, $4+4$; initial prostate-specific antigen, $11 \mathrm{ng} / \mathrm{mL}$ ), who received consecutive treatment with androgen deprivation therapy. At presentation of BCR, baseline PSMA PET showed some PSMA-avid lesions. (B) After additional treatment, scan revealed several new lesions. According to PPP, more than 2 new PSMA-avid lesions-and accordingly progressionare present.

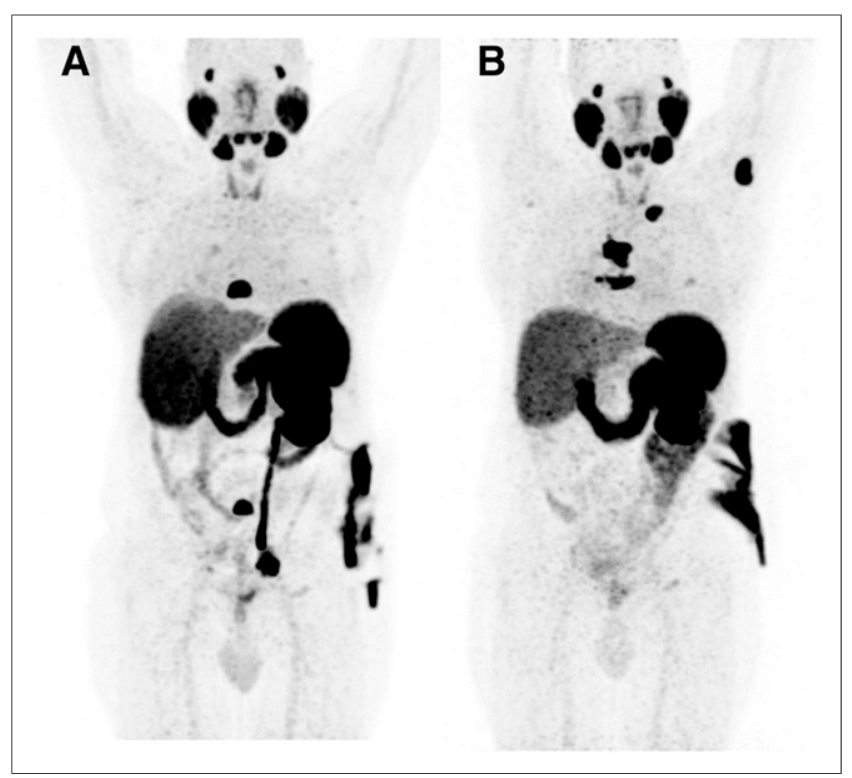

FIGURE 2. PPP for appearance of more than 2 new distant lesions, with other lesion disappearing, in 63-y-old patient with history of previous nephrectomy due to clear cell renal cell carcinoma. In 2011, patient underwent radical prostatectomy (pT3bpNO Mx; Gleason score, $4+5$; initial prostate-specific antigen, $11 \mathrm{ng} / \mathrm{mL}$ ) plus adjuvant external-beam radiation therapy. In 2016, patient experienced biochemical recurrence, which was treated with androgen deprivation therapy. (A) Castration resistance was diagnosed in 2018, with evidence of PSMA-positive bone lesions at dorsal and lumbar spine. (B) After external-beam radiation therapy to these lesions, previously evident lesions disappeared almost completely, but other new lesions appeared. Because there were more than 2 new PSMA-positive lesions, progression was diagnosed according to PPP.

mo of PSMA PET (by bone scintigraphy, MRI, or CT) is recommended but not required. In fact, false-positives are highly unlikely, as the known causes are usually easily identified by cross-sectional imaging.

Defining progression in the absence of new lesions by mere growth of an existing lesion remains challenging. Despite their limitations, the morphologic RECIST (10) are quite reliable for response assessment. Similarly, PERCIST (6) and the response criteria of the European Organisation for Research and Treatment of Cancer (11) for ${ }^{18}$ F-FDG PET imaging are robust and use defined changes in lesion SUV. However, because semiquantitative values are not established for PSMA PET, these response approaches cannot be directly translated into PSMA PET imaging (12). PPP aims to reliably identify disease progression while avoiding false-positives. Therefore, progression without new lesions is defined as increases in size or tracer uptake by at least $30 \%$. Findings should be confirmed by either biopsy or correlative imaging within 3 mo of PSMA PET. The cutoff of at least $30 \%$ is arbitrarily chosen and requires confirmation or adaptation in future prospective studies.

In summary, PPP defines PSMA PET-derived progression as 3 particular constellations: the appearance of 2 or more new PSMApositive distant lesions; the appearance of 1 new PSMA-positive lesion plus consistent clinical or laboratory data and recommended confirmation by biopsy or correlative imaging within 3 mo of PSMA PET; and an increase by at least $30 \%$ in size or uptake plus consistent clinical or laboratory data and confirmation by biopsy or correlative imaging within 3 mo of PSMA PET. 


\section{DISTANT VERSUS LOCAL PROGRESSION}

For the purpose of clinical trials, but also for management of patients, it is important to distinguish between distant and local progression. Metastasis-free survival has been demonstrated to correlate with survival in men with localized disease (13). Although the appearance of at least 1 new distant lesion is indicative of distant progression, the definition and clinical impact of local progression are less straightforward and require additional considerations.

Isolated local progression is quite uncommon, as most PSMA scans after treatment are performed on patients who have already undergone local treatment such as radical prostatectomy, definite or salvage radiation therapy, or nonconventional primary therapies. However, isolated local progression can occur and may include progression in the prostate or prostate bed, local recurrence (growth of existing lesion), or progression in local (pelvic) lymph nodes alone without any distant progression (a new local lesion). These situations are rare in our experience but nonetheless can occur and can be encompassed in the previously detailed definition. However, the progression is only local: whether local progression might be locally treated without requiring discontinuation of an otherwise successful therapy is a matter of debate.

\section{SPECIAL CONSIDERATIONS ABOUT DIFFERENT THERAPIES}

The proposed criteria can be applied for response assessment after any therapy, including locoregional and systemic treatments. Appropriate timing could be relevant, as is the case when the response to ${ }^{18} \mathrm{~F}-\mathrm{FDG}$ PET-based treatment is assessed. For instance,

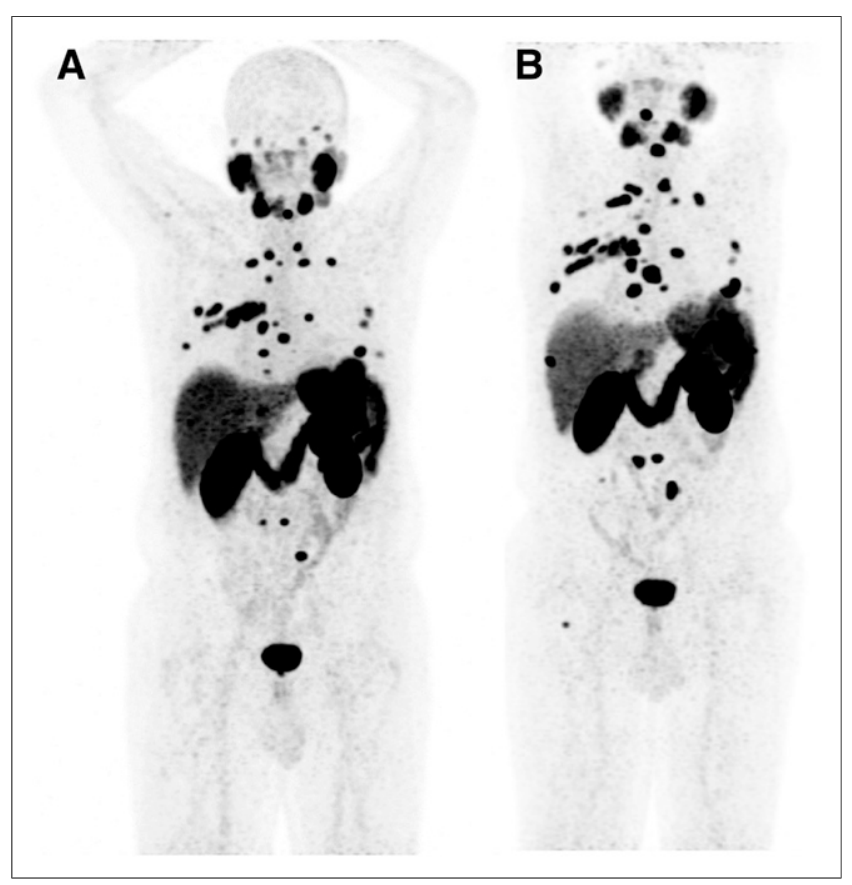

FIGURE 3. PPP for appearance of more than 2 new distant lesions in 70 -y-old patient who had radical prostatectomy (Gleason score, $5+4$; initial prostate-specific antigen, $7 \mathrm{ng} / \mathrm{mL}$ ) plus adjuvant external-beam radiation therapy in 2009. In 2013, biochemical recurrence was treated with lymphadenectomy plus androgen deprivation therapy. (A) Castration resistance occurred in 2017, with evidence of multiple bone lesions on PSMA PET. (B) After enzalutamide treatment, previously evident lesions were persistent, with appearance of a few new lesions fulfilling criteria for progression according to PPP.

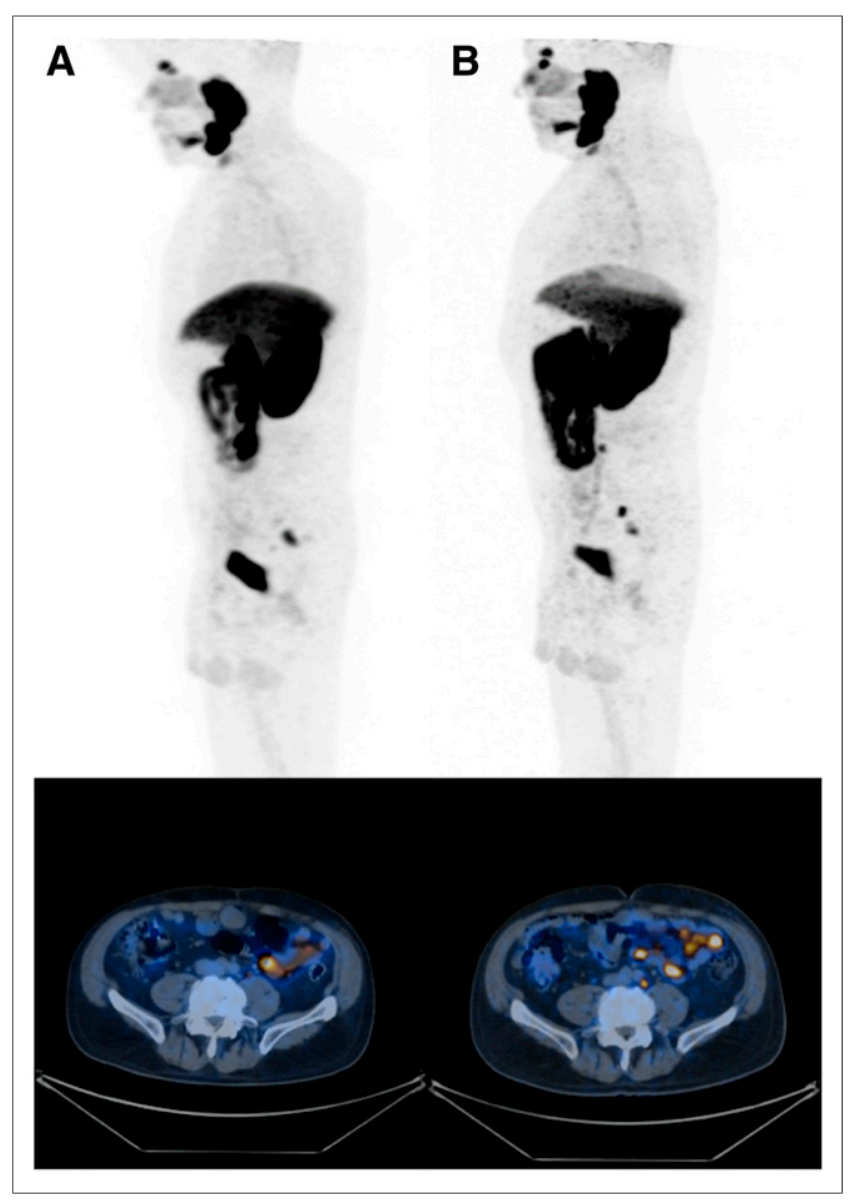

FIGURE 4. PPP for appearance of only 1 new distant lesion in 67-y-old patient with history of prostate cancer after radical prostatectomy and adjuvant external-beam radiation therapy in 2003. In 2015, biochemical recurrence was treated with salvage lymphadenectomy and androgen deprivation therapy. (A) Further increase in PSA occurred, with evidence of 1 nodal lesion on PSMA PET. (B) At follow-up, only 1 new nodal lesion appeared. According to PPP, in cases of only 1 new lesion, progression is confirmed only if there are consistent clinical or laboratory data and confirmation by biopsy or correlative imaging within 3 mo of PSMA PET.

intervals of 4 and $8 \mathrm{wk}$ are recommended for response assessment after chemotherapy and radiation therapy, respectively. Even longer intervals may be required after surgery. The proposed intervals are frequently applied in clinical routine despite the lack of prospective data; indeed, the intervals required for reliable response monitoring using PSMA PET imaging are unknown.

As shown in animal experimental and human studies, androgen deprivation therapy can result in PSMA overexpression, which may lead to false-positive findings (14). Alternatively, it may increase the number of true-positive findings (15). In most studies, the impact of androgen deprivation therapy and androgen receptor-targeted therapies on PSMA expression is more relevant early after the start of treatment (16). Therefore, PSMA PET response assessment of androgen deprivation therapy should probably be done 4-8 wk, at the earliest, after the start of treatment. Limited reports are available on the use of PSMA PET to evaluate radioligand therapy responses (e.g., with ${ }^{177}$ Lu-PSMA-617). Preliminary data suggest that PPP could be applicable in this setting also (17). 


\section{CONFIRMATORY TRIAL}

The PPP proposal is not evidence-based but rather an initial motivational push to arrive at a structured response assessment. Thus, the current proposal can be only a starting point for a validation study that will hopefully arrive at robust response and progression criteria. We therefore propose an international data repository that includes all prospective treatment trials that use PSMA PET assessment of metastatic prostate cancer. These data can then be used to validate the PPP performance against histopathology or correlative imaging within 3 mo of PSMA PET. A correlation between PPP assessment and survival is of interest but might be of limited value because of the bias of many consecutive treatments.

\section{DISCUSSION}

Morphologic response criteria such as RECIST used to be the backbone of treatment response assessment in metastatic prostate cancer. This changed with the introduction of PCWG2, which based progression mainly on bone scan findings. The appearance of 2 or more new lesions, which in cases of ambiguity had to be confirmed by other imaging modalities, was required for progression. PCWG2 recommended bone scans and conventional imaging (CT or MRI) in addition to blood work and symptom assessment (both every cycle) at 12-wk intervals. The PCWG3 update, published in 2016, did not significantly change the image-based definition of disease progression (2). The PCWG2 criteria proved to be reliable endpoints and were prognostic for overall survival and radiographic progression-free survival within clinical drug development trials $(18,19)$.

With the introduction of ligands targeting PSMA (20), PET has changed prostate cancer imaging. Within only $5 \mathrm{y}$, PSMA imaging is now ubiquitously available and under consideration for Food and Drug Administration approval. It also has been incorporated into major clinical guidelines for detection of biochemical recurrence at very low levels of serum prostate-specific antigen (European Association of Urology guidelines). The confirmed high positive predictive value (21) and higher sensitivity for disease detection (4) also suggest superiority for assessing disease progression. However, the available data preclude the establishment of evidence-based PPP criteria. There is a need for well-defined progression criteria as patients, their families, and their physicians request unequivocal diagnostic information from PSMA PET studies. Moreover, drug companies are integrating PSMA imaging into clinical trials and thus require firm definitions of progressive disease.

Here, we propose a pathway to addressing this need by developing PPP criteria based on PCWG2 criteria (Table 1). We also propose a strategy to validate or adjust the criteria by creating a repository that would include data from any prospective clinical trials that use PSMA imaging for response assessment. Finally, PPP will also attempt to derive prognostic information on patients with progressive disease because PPP distinguishes between distant and local progression.

Identification of disease progression is essential in clinical practice and in trials to determine the effectiveness of an established or new therapy. The overarching goal of therapy is improved patient outcome. The fact that PPP focuses on distant disease may add granularity to prognostication. PPP criteria may be more sensitive than the established PCWG2 and PCWG3 criteria because progression is assessed earlier. We are aware that high specificity is equal in importance to sensitivity, because false-positives may result in overtreatment. We attempted to address this issue by requiring histopathology and image verification in patients with only 1 new distant lesion or growing lesions on PSMA scans.

Because PSMA PET is significantly more sensitive but also more specific than bone scans, the single presentation of 2 new lesions is sufficient for progression. False-positive PSMA findings are much less frequent than false-positive bone scan findings (9). Moreover, they rarely appear in 2 locations at the same time. PPP also considers a single new distant lesion as progression, if the finding is consistent with the clinical presentation. This consideration is justified because PSMA imaging is highly sensitive and specific and lesion verification by biopsy, conventional imaging, or laboratory findings is required. According to PPP, an increase in size and PSMA uptake of at least $30 \%$ is also coupled with disease progression. Reproducibility studies for PSMA PET have suggested that such changes are beyond the expected reproducibility or repeatability variance.

Thus far, PSMA PET has been validated in patients with biochemical recurrence and in the setting of restaging (21). In contrast, its role for disease staging and therapy response monitoring is unknown. It may be argued that using PET for evaluating clinical outcomes in advanced prostate cancer may not be cost-effective. However effective patient care and efficient drug development require accurate tools to assess treatment effects. For metastatic prostate cancer, response biomarkers have historically been poorly reproducible, inaccurate, inconsistently applied, or only loosely associated with tangible clinical benefits such as survival (22). Furthermore, available treatments are quite expensive, and reliable intermediate endpoint biomarkers are needed to identify nonresponders early after the start of therapy. Also, in patients undergoing androgen deprivation therapy, PSMA PET imaging might be helpful.

Studies assessing the role of different PSMA PET response criteria are lacking. A recent paper by Gupta et al. (12) compared several criteria (RECIST, PERCIST, European Organisation for

TABLE 1

PPP Criteria

\begin{tabular}{cc}
\hline \multicolumn{1}{c}{ Progression criterion } & Explanation \\
\hline $\begin{array}{l}2 \text { or more new PSMA-positive } \\
\text { lesions }\end{array}$ & Appearance of 2 or more new PSMA-positive distant lesions \\
$\begin{array}{l}1 \text { new PSMA-positive } \\
\text { lesion }\end{array}$ & $\begin{array}{c}\text { Appearance of } 1 \text { new PSMA-positive lesion plus consistent clinical or laboratory } \\
\text { data and recommended confirmation by biopsy or correlative imaging within } 3 \text { mo of PSMA PET }\end{array}$ \\
$\begin{array}{l}\text { No new lesions but size } \\
\text { increase }\end{array}$ & $\begin{array}{c}\text { Increase by } \geq 30 \% \text { in size or uptake plus consistent clinical or laboratory data and } \\
\text { confirmation by biopsy or correlative imaging within } 3 \text { mo of PSMA PET }\end{array}$
\end{tabular}


Research and Treatment of Cancer, and M.D. Anderson) for assessing treatment response with ${ }^{68} \mathrm{Ga}$-PSMA PET/CT in patients who have metastatic prostate cancer with biochemical progression. The paper concluded that molecular criteria performed better than morphologic parameters. This conclusion is expected, as functional approaches provide earlier response information than anatomic imaging methods. The PPP criteria we are proposing are exquisitely functional and may provide early — and highly clinically relevantinformation on therapy response.

We are aware that PPP is only the beginning of standardized PSMA-driven response assessment in patients with metastatic prostate cancer. For the future evolution of PPP, several determinants have to be closely monitored, including the variety and combination of therapies and the consecutively different impact on the PSMA signal $(16,23,24)$; the so-far arbitrarily chosen discriminator of at least a $30 \%$ SUV reduction, disregarding volume parameters; the still not fully understood influence of androgen-receptor-targeting therapies on PSMA expression (25); and the possibility that another complementary metabolic tracer such as ${ }^{18}$ F-FDG will be needed. However, we believe this is an excellent starting point and warrants further evaluation, testing, and discussion.

\section{DISCLOSURE}

Stefano Fanti receives personal fees (sponsored lectures, advisory board participation) or nonfinancial support (congress attendance) from AAA, ANMI, Astellas, Bayer, BED, GE Healthcare, IBA, Janssen, and Sanofi (all outside the submitted work). Boris Hadaschik receives grants, personal fees, and nonfinancial support from Janssen; grants from German Cancer Aid, German Research Foundation, and Profound Medical; personal fees and nonfinancial support from Astellas, Bayer, BMS, Lightpoint Medical, Astra Zeneca, and Sanofi; and grants and personal fees from Uromed (all outside the submitted work). Ken Herrmann receives personal fees from Bayer, other stock options from Sofie Biosciences, personal fees from SIRTEX, other free research material from $\mathrm{ABX}$, personal fees and nonfinancial support from Adacap, personal fees from Curium, personal fees from Endocyte, grants and personal fees from BTG, personal fees from IPSEN, personal fees and nonfinancial support from Siemens Healthineers, nonfinancial support from GE Healthcare, personal fees from Astellas, and personal fees from yMabs (all outside the submitted work). No other potential conflict of interest relevant to this article was reported.

\section{REFERENCES}

1. Scher HI, Halabi S, Tannock I, et al. Design and end points of clinical trials for patients with progressive prostate cancer and castrate levels of testosterone: recommendations of the Prostate Cancer Clinical Trials Working Group. J Clin Oncol. 2008;26:1148-1159.

2. Scher HI, Morris MJ, Stadler WM, et al. Trial design and objectives for castration-resistant prostate cancer: updated recommendations from the Prostate Cancer Clinical Trials Working Group 3. J Clin Oncol. 2016;34:1402-1418.

3. Han S, Woo S, Kim YJ, Suh CH. Impact of ${ }^{68} \mathrm{Ga}$-PSMA PET on the management of patients with prostate cancer: a systematic review and meta-analysis. Eur Urol. 2018;74:179-190.

4. Perera M, Papa N, Roberts M, et al. Gallium-68 prostate-specific membrane antigen positron emission tomography in advanced prostate cancer-updated diagnostic utility, sensitivity, specificity, and distribution of prostate-specific membrane antigen-avid lesions: a systematic review and meta-analysis. Eur Urol. February 14, 2019 [Epub ahead of print].
5. Werner RA, Bundschuh RA, Bundschuh L, et al. Novel structured reporting systems for theranostic radiotracers. J Nucl Med. 2019;60:577-584.

6. Eiber M, Herrmann K, Calais J, et al. Prostate Cancer Molecular Imaging Standardized Evaluation (PROMISE): proposed miTNM classification for the interpretation of PSMA-ligand PET/CT. J Nucl Med. 2018;59:469-478.

7. Fanti S, Minozzi S, Morigi JJ, et al. Development of standardized image interpretation for ${ }^{68} \mathrm{Ga}$-PSMA PET/CT to detect prostate cancer recurrent lesions. Eur J Nucl Med Mol Imaging. 2017;44:1622-1635.

8. Rowe SP, Pienta KJ, Pomper MG, Gorin MA. Proposal for a structured reporting system for prostate-specific membrane antigen-targeted PET imaging: PSMARADS version 1.0. J Nucl Med. 2018;59:479-485.

9. Malik D, Sood A, Mittal BR, et al. Nonspecific uptake of ${ }^{68} \mathrm{Ga}$-prostate-specific membrane antigen in diseases other than prostate malignancy on positron emission tomography/computed tomography imaging: a pictorial assay and review of literature. Indian J Nucl Med. 2018;33:317-325.

10. Eisenhauer EA, Therasse P, Bogaerts J, et al. New response evaluation criteria in solid tumours: revised RECIST guideline (version 1.1). Eur J Cancer. 2009;45:228-247.

11. Young H, Baum R, Cremerius U, et al. Measurement of clinical and subclinical tumour response using $\left[{ }^{18} \mathrm{~F}\right]$-fluorodeoxyglucose and positron emission tomography: review and 1999 EORTC recommendations. European Organization for Research and Treatment of Cancer (EORTC) PET Study Group. Eur J Cancer. 1999;35:1773-1782.

12. Gupta M, Choudhury PS, Rawal S, Goel HC, Rao SA. Evaluation of RECIST, PERCIST, EORTC, and MDA criteria for assessing treatment response with Ga68-PSMA PET-CT in metastatic prostate cancer patient with biochemical progression: a comparative study. Nucl Med Mol Imaging. 2018;52:420-429.

13. Xie W, Regan MM, Buyse M, et al. Metastasis-free survival is a strong surrogate of overall survival in localized prostate cancer. J Clin Oncol. 2017;35:3097-3104.

14. Aggarwal R, Wei X, Kim W, et al. Heterogeneous flare in prostate-specific membrane antigen positron emission tomography tracer uptake with initiation of androgen pathway blockade in metastatic prostate cancer. Eur Urol Oncol. 2018;1:7882.

15. Lückerath K, Wei L, Fendler WP, et al. Preclinical evaluation of PSMA expression in response to androgen receptor blockade for theranostics in prostate cancer. EJNMMI Res. 2018;8:96.

16. Emmett L, Yin C, Crumbaker M, et al. Rapid modulation of PSMA expression by androgen deprivation: serial ${ }^{68} \mathrm{Ga}$-PSMA-11 PET in men with hormone-sensitive and castrate-resistant prostate cancer commencing androgen blockade. J Nucl Med. 2019;60:950-954.

17. Emmett L, Crumbaker M, Ho B, et al. Results of a prospective phase 2 pilot trial of ${ }^{177} \mathrm{Lu}-\mathrm{PSMA}-617$ therapy for metastatic castration-resistant prostate cancer including imaging predictors of treatment response and patterns of progression. Clin Genitourin Cancer. 2019;17:15-22.

18. Rathkopf DE, Beer TM, Loriot Y, et al. Radiographic progression-free survival as a clinically meaningful end point in metastatic castration-resistant prostate cancer: the PREVAIL randomized clinical trial. JAMA Oncol. 2018;4:694-701.

19. Sonpavde G, Pond GR, Armstrong AJ, et al. Radiographic progression by Prostate Cancer Working Group (PCWG)-2 criteria as an intermediate endpoint for drug development in metastatic castration-resistant prostate cancer. BJU Int. 2014;114:E25E31.

20. Afshar-Oromieh A, Malcher A, Eder M, et al. PET imaging with a $\left[{ }^{68} \mathrm{Ga}\right]$ galliumlabelled PSMA ligand for the diagnosis of prostate cancer: biodistribution in humans and first evaluation of tumour lesions. Eur J Nucl Med Mol Imaging. 2013;40:486-495.

21. Fendler WP, Calais J, Eiber M, et al. Assessment of ${ }^{68}$ Ga-PSMA-11 PET accuracy in localizing recurrent prostate cancer: a prospective single-arm clinical trial. JAMA Oncol. 2019;5:856-863.

22. Morris MJ, Autio KA, Basch EM, Danila DC, Larson S, Scher HI. Monitoring the clinical outcomes in advanced prostate cancer: what imaging modalities and other markers are reliable? Semin Oncol. 2013;40:375-392.

23. Sathekge M, Bruchertseifer F, Vorster M, et al. Predictors of overall and diseasefree survival in metastatic castration-resistant prostate cancer patients receiving ${ }^{225}$ Ac-PSMA-617 radioligand therapy. J Nucl Med. 2020;61:62-69.

24. Seitz AK, Rauscher I, Haller B, et al. Preliminary results on response assessment using ${ }^{68} \mathrm{Ga}-\mathrm{HBED}-\mathrm{CC}-\mathrm{PSM} \mathrm{PET} / \mathrm{CT}$ in patients with metastatic prostate cancer undergoing docetaxel chemotherapy. Eur J Nucl Med Mol Imaging. 2018;45:602-612.

25. Hope TA, Truillet C, Ehman EC, et al. ${ }^{68} \mathrm{Ga}-\mathrm{PSMA}-11$ PET imaging of response to androgen receptor inhibition: first human experience. $J$ Nucl Med. 2017;58:8184. 